\title{
BROOKHGUEN
}

NATIONAL LABORATORY

BNL-112143-2016-JA

\section{Identification of Microscopic Hole-trapping Mechanisms in Nitride Semiconductorsace}

\author{
John L. Lyons \\ John L. Lyons, Karthik Krishnaswamy, Luke Gordon, Anderson Janotti, \\ and Chris G. Van de Walle
}

Submitted to the Journal of IEEE ELECTRON DEVICE LETTERS

Center for Functional Nanomaterials

Brookhaven National Laboratory

\author{
U.S. Department of Energy \\ Office of Basic Energy Sciences
}

\footnotetext{
Notice: This manuscript has been authored by employees of Brookhaven Science Associates, LLC under Contract No. DE- SC0012704 with the U.S. Department of Energy. The publisher by accepting the manuscript for publication acknowledges that the United States Government retains a non-exclusive, paid-up, irrevocable, world-wide license to publish or reproduce the published form of this manuscript, or allow others to do so, for United States Government purposes.
} 


\section{DISCLAIMER}

This report was prepared as an account of work sponsored by an agency of the United States Government. Neither the United States Government nor any agency thereof, nor any of their employees, nor any of their contractors, subcontractors, or their employees, makes any warranty, express or implied, or assumes any legal liability or responsibility for the accuracy, completeness, or any third party's use or the results of such use of any information, apparatus, product, or process disclosed, or represents that its use would not infringe privately owned rights. Reference herein to any specific commercial product, process, or service by trade name, trademark, manufacturer, or otherwise, does not necessarily constitute or imply its endorsement, recommendation, or favoring by the United States Government or any agency thereof or its contractors or subcontractors. The views and opinions of authors expressed herein do not necessarily state or reflect those of the United States Government or any agency thereof. 


\title{
Identification of Microscopic Hole-trapping Mechanisms in Nitride Semiconductors
}

\author{
John L. Lyons, Karthik Krishnaswamy, Luke Gordon, Anderson Janotti, and Chris G. Van de Walle
}

\begin{abstract}
Hole trapping has been observed in nitride heterostructure devices where the Fermi level is in the vicinity of the valence-band maximum. Using hybrid density functional calculations, we examine microscopic mechanisms for hole trapping in GaN and AlN. In defect-free material, hole trapping does not spontaneously occur, but trapping can occur in the vicinity of impurities such as $\mathbf{C}-\mathbf{a}$ common unintentional impurity in nitrides. Using Schrödinger-Poisson simulations, we assess the effects of $\mathrm{C}$-derived hole traps on $\mathrm{N}$-face high-electron mobility transistors, which we find to be more detrimental than the previously proposed interface traps.
\end{abstract}

Index Terms-Hole traps, nitride semiconductors, firstprinciples calculations, impurities.

\section{INTRODUCTION}

A number of nitride semiconductor device designs employ heterostructures in which the Fermi level approaches the valence-band maximum (VBM) due to the effect of polarization fields. One case is a class of devices in which a polarization-induced hole gas is engineered to arise in a graded AlGaN alloy [1]. Other examples occur in $\mathrm{N}$ face high-electron-mobility transistors (HEMTs), in which a two-dimensional hole gas (2DHG) should be present at the interface between the AlGaN back barrier and $\mathrm{GaN}$ buffer [2], and metal-face $p$-channel field-effect transistors, in which polarization engineering also leads to 2DHGs [3], [4]. A final example is interband tunneling junctions, in which trapping has been observed at interfaces [5]. In each of these heterostructures, the presence of hole traps has been found to adversely affect device performance. In the polarization-holedoped heterostructures, such hole traps are thought to prevent the formation of free holes [1]. In N-face HEMTs, the hole traps have been proposed to cause large-signal dispersion that is observed in current-voltage curves [2], [6], and in tunnel junctions, such traps have been blamed for decreasing tunneling current [5].

Based on deep level transient spectroscopy, a donor-like defect with a transition level $60 \mathrm{meV}$ from the VBM of GaN

This work was supported by the Center for Low Energy Systems Technology (LEAST), one of the six SRC STARnet Centers, sponsored by MARCO and DARPA, and by the National Science Foundation (NSF) under grant No. DMR-1434854. The work made use of the Center for Scientific Computing at the CNSI and MRL: an NSF MRSEC (DMR-1121053) and NSF CNS-0960316, as well as the Extreme Science and Engineering Discovery Environment (XSEDE), supported by NSF (OCI-1053575 and NSF DMR070072N).

We thank Dr. S. Keller and Prof. S. Rajan for helpful discussions. All authors are with the Materials Department of University of California, Santa Barbara, Santa Barbara, CA, 93106-5050 USA. Contact address: Center for Functional Nanomaterials, Brookhaven National Laboratories, Upton, NY 11973, e-mail: (jlyons@bnl.gov). was proposed to be responsible for hole-trapping behavior [2], [6]. Dispersion in the CV curves was suppressed with $\mathrm{Si}$ doping of the GaN buffer [6], as this $n$-type doping establishes the Fermi level far away from the VBM. However, the microscopic mechanism whereby the hole trapping occurs has not yet been established.

Here we examine possible hole-trapping mechanisms using hybrid density functional calculations. We find that selftrapping of holes does not occur in bulk material but can occur in the presence of impurities. We focus on $\mathrm{C}$, an impurity almost always present in the nitrides due to unintentional incorporation. Using Schrödinger-Poisson (SP) simulations we examine the effect on $\mathrm{N}$-face HEMTs of $\mathrm{C}$ hole traps distributed throughout the $\mathrm{GaN}$ layer, and compare this behavior with that of the interface traps that have been previously proposed. The distributed hole trap is found to deplete the hole gas at the $\mathrm{AlGaN} / \mathrm{GaN}$ back barrier interface in a fashion that is virtually indistinguishable from the interface traps, supporting our proposal that distributed $\mathrm{C}$ impurities are the cause of hole trapping in the heterostructures.

\section{Methods}

We employ first-principles calculations based on the KohnSham scheme [8] with the HSE hybrid functional [9] as implemented within VASP [10]. The use of the hybrid functional is necessary for an accurate description of the semiconductor band gap and for correctly describing hole localization at defects and impurities [7]. Calculations for trapped holes are carried out in 96-atom supercells; other details of the calculations are as in Ref. [7]. The SP simulations were carried out using the nextnano ${ }^{3}$ code [11].

\section{RESUlts AND Discussion}

First-principles calculations of hole-trapping mechanisms. We first examine whether holes self-trap in bulk material (also known as small-polaron formation). In bulk GaN, we previously found that holes do not self-trap [12]. We have also examined whether a hole would self-trap in the presence of biaxial strain in pseudomorphic layers. Imposing a $2 \%$ biaxial strain does not alter the stability of the delocalized hole (the spin density remains similar to the unstrained case) with no tendency for hole localization. We also examined whether the presence of $\mathrm{Al}$ could lead to hole trapping in $\mathrm{GaN}$ by placing a single isoelectronic cation in the $\mathrm{GaN}$ supercell. Again, no self-trapping occurs. All of this is reassuring from the point of view of hole transport in $\mathrm{GaN}$, for which hole self-trapping would be detrimental. 


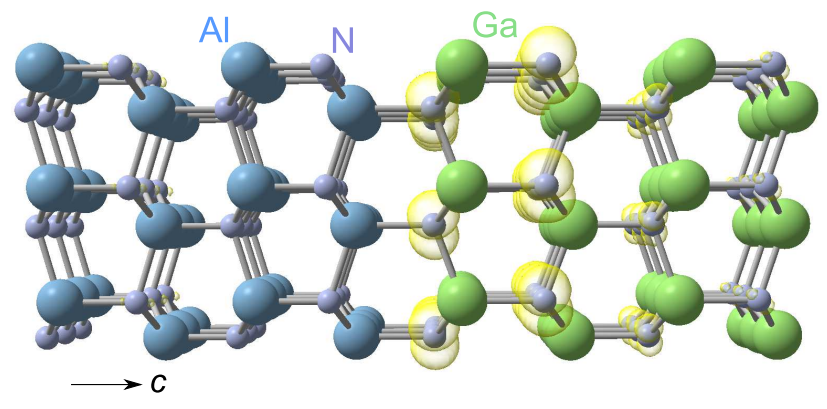

Fig. 1. Spin densities a delocalized hole at a GaN/AlN interface.

A self-trapped hole can be stabilized in bulk AlN. Localization occurs mainly on a single $\mathrm{N}$ atom, accompanied by an increase in the bond length of the axial Al-N bond by $13 \%$. However, the hole polaron is metastable, since the delocalized hole is lower in energy (by $0.30 \mathrm{eV}$ ) than the self-trapped hole. In equilibrium, we thus do not expect holes to self-trap in AlN. As for GaN, we also found that neither biaxial strain nor the presence of Ga leads to a trapped hole that is more stable than the delocalized hole.

To investigate whether holes might trap at an GaN/AlN interface, we constructed a GaN/AlN superlattice along the [0001] direction with 4 bilayers each of $\mathrm{GaN}$ and AlN, using the in-plane lattice constant of $\mathrm{GaN}$ and a $(2 \times 2)$ in-plane periodicity to allow for symmetry-breaking relaxations [Fig. 1]. A number of different distortions were explored, but none resulted in hole localization. Every structure tested relaxed into the delocalized-hole configuration shown in Fig. 1. The hole remains delocalized in the GaN part of the superlattice, as expected based on the valence-band offset between $\mathrm{GaN}$ and AlN, but near the [0001] end of the layer, consistent with the presence of polarization fields. Since the tendency for hole trapping should be strongest in AlN, as it has the highest hole mass and lowest-lying valence band, our results indicate that hole trapping will also not occur at $\mathrm{AlGaN} / \mathrm{GaN}$ interfaces.

Self-trapping of holes will therefore not occur in pristine material or near interfaces. Yet hole polarons may be stabilized at impurities [7]. In Fig. 2 we list candidate hole-trapping centers and their charge-state transition levels in GaN. Each of these impurities (or complexes) nominally acts as an acceptor [associated with a (0/-) level], but (with the exception of $\left.\mathrm{Mg}_{\mathrm{Ga}}\right)$ they also exhibit a (+/0) level near the VBM. Such a level is usually associated with donors, but due to its proximity to the VBM here it indicates that the impurity acts as a hole trap when the Fermi level is low in the gap.

$\mathrm{Mg}_{\mathrm{Ga}}$ does not give rise to a hole trap by itself, but it can form a complex with hydrogen. $\mathrm{Mg}-\mathrm{H}$ complexes are usually assumed to be electrically neutral (and need to be dissociated before $\mathrm{Mg}_{\mathrm{Ga}}$ can become electrically active). However, we have found that $\mathrm{Mg}-\mathrm{H}$ does exhibit a (+/0) hole-trapping level $130 \mathrm{meV}$ above the GaN VBM [13]. Such complexes could be present if intentional doping is performed in other regions of the heterostructure. Unintentional contamination with $\mathrm{Mg}$ (and formation of $\mathrm{Mg}-\mathrm{H}$ complexes) could also occur if memory effects are present in a deposition system in which $\mathrm{Mg}$-doped layers have previously been grown. Since the position of the

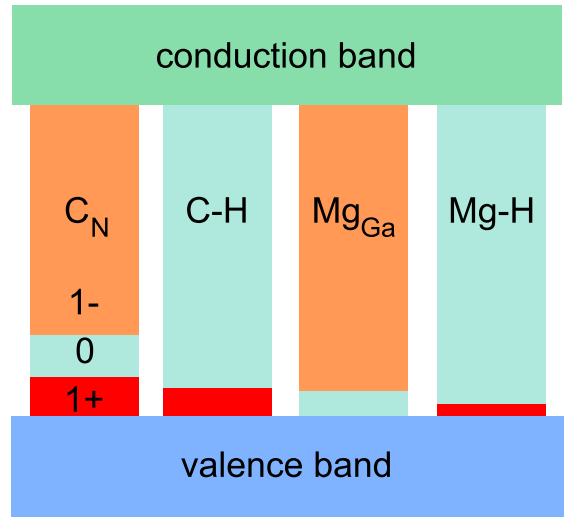

Fig. 2. Charge-state transition levels and charge-state regions of stability for acceptor impurities in GaN. Stability regions are color-coded by charge state as labeled for $\mathrm{C}_{\mathrm{N}}$ : when the Fermi level is in the orange region, the - charge state is stable; blue: neutral; and red:.$+ \mathrm{Mg}_{\mathrm{Ga}}$ has a $(0 /-)$ transition level as expected for an acceptor. The other impurities and complexes also exhibit a $(+/ 0)$ level near the VBM, turning them into hole traps.

$(+/ 0)$ level in the $\mathrm{Mg}-\mathrm{H}$ complex is similar to $\mathrm{C}_{\mathrm{N}}$, much of the discussion below will also apply to this complex.

The remainder of our discussion will focus on $\mathrm{C}$, a common unintentional impurity in GaN and AlN. $\mathrm{C}_{\mathrm{N}}$ acts as a deep acceptor, with a (0/-) level at $0.90 \mathrm{eV}$ above the $\mathrm{VBM}$ in $\mathrm{GaN}$ and $1.88 \mathrm{eV}$ in $\mathrm{AlN}$, but also features a (+/0) transition level at $0.35 \mathrm{eV}$ above the VBM in GaN and at $1.07 \mathrm{eV}$ above the VBM in AlN [7]. $\mathrm{C}_{\mathrm{N}}$ is the most stable form of the carbon impurity in both materials under most Fermi-level conditions, and is thus likely to be the dominant form of $\mathrm{C}$ in unintentionally doped material. Similar to the case of $\mathrm{Mg}$ acceptors, a C-H complex can also form when $\mathrm{H}$ is present. This complex features a (+/0) transition level at $0.25 \mathrm{eV}$ above the VBM in GaN [Fig. 2].

Schrödinger-Poisson simulations of heterostructures. We now examine the effect of hole traps on nitride devices by performing Schrödinger-Poisson simulations. We use the Nface HEMT device structure shown in Fig. 3 (similar to the one investigated in [5]), which contains a $30 \mathrm{~nm} \mathrm{GaN}$ channel, $30 \mathrm{~nm}$ of $\mathrm{Al}_{0.3} \mathrm{Ga}_{0.7} \mathrm{~N}$ back barrier, and a $100 \mathrm{~nm} \mathrm{GaN}$ buffer layer. Under zero bias this device exhibits a two-dimensional electron gas (2DEG) at the GaN channel/AlGaN interface and a $2 \mathrm{DHG}$ at the $\mathrm{AlGaN} / \mathrm{GaN}$ buffer interface where the valence bands of GaN approaches the Fermi level (at zero energy on the left $y$-axis).

To investigate hole trapping in this HEMT we examine two cases. Case 1 reflects the proposal [2], [6] that hole traps at 60 $\mathrm{meV}$ above the VBM are present in a 0.2 -nm-wide region at the $\mathrm{AlGaN} / \mathrm{GaN}$ buffer interface, with a sheet density between 0.1 and $1.5 \times 10^{12} \mathrm{~cm}^{-2}$ (note that such a high interface trap density is unlikely to be caused by unintentional impurities; a 1-nm-wide region would mean a volume trap density of at least $\left.1 \times 10^{18} \mathrm{~cm}^{-3}\right)$. In Case 2 we examine hole traps with a $(+/ 0)$ level at $350 \mathrm{meV}$ above the VBM (as for $\mathrm{C}_{\mathrm{N}}$ ) distributed throughout the $\mathrm{GaN}$ buffer region with a density between 0.1 and $1.5 \times 10^{17} \mathrm{~cm}^{-3}$; these $\mathrm{C}$ densities were chosen to reflect a realistic level of unintentional incorporation. We have also explored the presence of $\mathrm{C}$ in the $\mathrm{AlGaN}$ back-barrier region, 


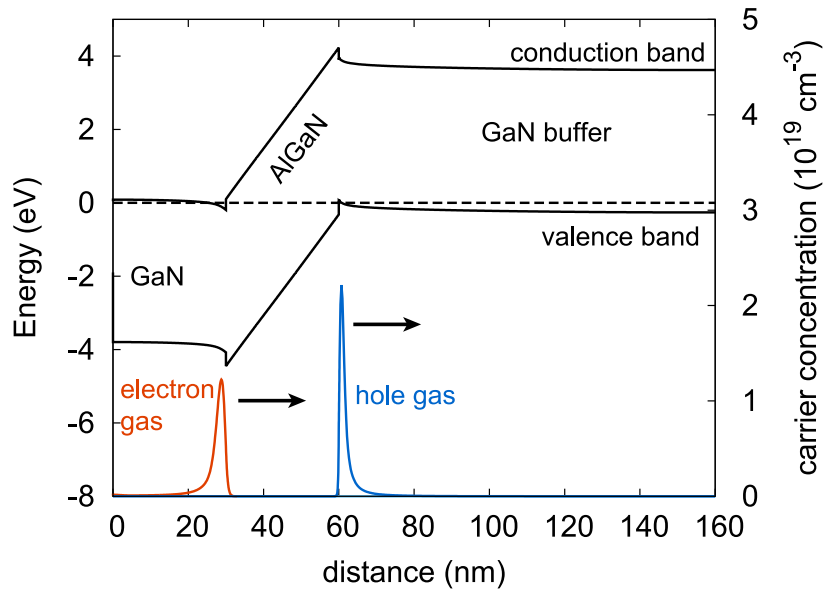

Fig. 3. Band structure of a N-face HEMT with a design based on Ref. [6], in which a $2 \mathrm{DHG}$ accumulates at the $\mathrm{AlGaN} / \mathrm{GaN}$ buffer interface (at $60 \mathrm{~nm}$ along the $x$-axis). The Fermi level is at zero energy (left axis), indicated by the dotted horizontal line. The carrier distributions in the 2DEG and 2DHG are also shown (right axis).

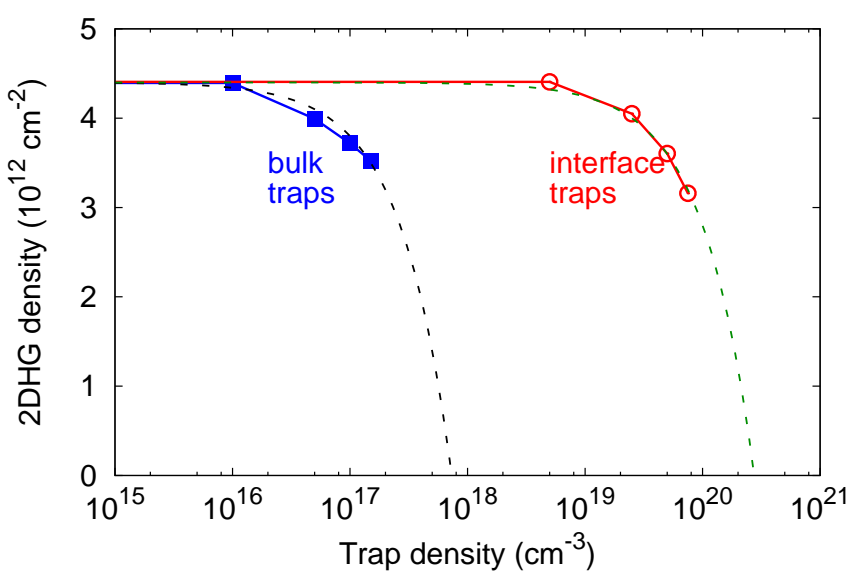

Fig. 4. 2DHG density versus trap density for the case of a $60-\mathrm{meV}$ hole trap located at the interface (Case 1, in red) and for the case of 350-meV C-related hole traps distributed in the GaN buffer (Case 2, in blue). Dotted lines indicate fits meant to provide estimates for carrier densities necessary for complete hole trapping.

but found it has a negligible effect on the 2DHG concentration.

The density of the 2DHG as a function of trap density for these two cases is shown in Fig. 4. In both cases, the traps reduce the density of the 2DHG: instead of polarization charges being compensated by free holes, holes are bound to the hole traps, and these positively charged centers compensate the polarization charge. In fact, Case 2 (C traps distributed throughout the $\mathrm{GaN}$ buffer) leads to a much greater reduction in the 2DHG density than the $60 \mathrm{meV}$ interface traps at equivalent trap density. As a reduction in 2 DHG density by donorlike traps has been linked to anomalous output conductance in $\mathrm{N}$-face nitride-based HEMTs, our results suggest that $\mathrm{C}$ impurities in the buffer are a better candidate for the hole traps affecting these devices.

These results indicate that impurities can act as hole traps within nitride heterostructures, and that contamination by $\mathrm{C}$ impurities or $\mathrm{Mg}-\mathrm{H}$ complexes should be avoided. Hydrogenation, often used to passivate traps, would be ineffective here: both $\mathrm{Mg}-\mathrm{H}$ and $\mathrm{C}-\mathrm{H}$ complexes exhibit (+/0) trapping levels near the GaN VBM (see Fig. 2) and thus act as holetrapping centers. Hydrogen is a ubiquitous impurity, present even in MBE growth environments. The concentration of hydrogen should thus be carefully monitored and controlled. In structures grown by metal-organic chemical vapor deposition where $\mathrm{Mg}$ doping is employed intentionally (as is the case, e.g., in Refs. [3] and [4]), post-growth annealing is performed in order to remove the hydrogen and activate the $\mathrm{Mg}$ acceptors. However, it is known that such an annealing procedure does not necessarily remove all of the hydrogen [14], and therefore some concentration of $\mathrm{Mg}-\mathrm{H}$ complexes may still be present. Our results indicate the importance of keeping the concentration of such complexes low, ideally below $10^{16} \mathrm{~cm}^{-3}$, because otherwise the 2DHG density may be affected (see Fig. 4). We note that, in structures of the type shown in Fig. 3, the electrostatics will inhibit the removal of hydrogen from the sample through the surface; structures with the $p$-type layer near the surface [3], [4] should therefore be favored.

\section{CONCLUSION}

Using hybrid density functional calculations we have investigated microscopic mechanisms for hole trapping in $\mathrm{GaN}$ and AlN. Self-trapped holes are not stable in GaN or AlN. However, holes can trap at impurity sites, and we propose unintentionally incorporated $\mathrm{C}$ (or $\mathrm{Mg}-\mathrm{H}$ complexes) as candidates for hole-trapping centers in nitride devices.

\section{REFERENCES}

[1] J. Simon, V. Protasenko, C. Lian, and D. Jena, "Polarization-induced hole doping in wide-band-gap uniaxial semiconductor heterostructures," Science, vol. 327, pp. 60-64, Jan. 2010.

[2] S. Rajan, A. Chini, M. H. Wong, J. S. Speck, and U. K. Mishra, "N-polar GaN/AlGaN/GaN high electron mobility transistors," J. Appl. Phys., vol. 102, no. 4, pp. 044501-1-044501-6, Aug. 2007.

[3] B. Reuters, H. Hahn, A. Pooth, B. Höllander, U. Breuer, M. Heuken, H. Kalisch, and A. Vescan, "Fabrication of p-channel heterstructure field effect transistors with polarization-induced two-dimensional hole gases at metal-polar GaN/AlInGaN interfaces," J. Phys. D: Appl. Phys., vol. 47, pp. 175103-1-175103-10, Apr. 2014.

[4] A. Nakajima, Y. Sumida, M. H. Dhyani, H. Kawai, and E. M. S. Narayanan, "High density two-dimensional hole gas induced by negative polarization at GaN/AlGaN heterointerface," J. Appl. Phys., vol. 102, no. 4, pp. 175103-1-175103-10, Apr. 2014.

[5] S. Krishnamoorthy, P. S. Park, and S. Rajan, "Demonstration of forward inter-band tunneling in GaN by polarization engineering," Appl. Phys. Lett., vol. 99, pp. 233504-1-233504-3, Dec. 2011.

[6] M. H. Wong, U. Singisetti, J. Lu, J. S. Speck, and U. K. Mishra, "Anomalous output conductance in N-polar GaN high electron mobility transistors," IEEE Trans. on Elec. Devices, vol. 59, no. 11, pp. 29882995, July 2012.

[7] J. L. Lyons, A. Janotti, and C. G. Van de Walle, "Effects of carbon on the electrical and optical properties of InN, GaN, and AlN," Phys. Rev. $B$, vol. 89, no. 3, pp. 035204-1-035204-8, Jan. 2014.

[8] W. Kohn and L. J. Sham, "Self-consistent equations including exchange and correlation effects," Phys. Rev. B, vol. 140, no. 4A, pp. A1133A1138, Nov. 1965.

[9] J. Heyd, G. E. Scuseria, and M. Ernzerhof, "Hybrid functionals based on a screened Coulomb potential," J. Chem. Phys., vol. 124, no. 21, pp. 219906, June 2006.

[10] G. Kresse and J. Furthmüller, "Efficient iterative schemes for $a b$ initio total-energy calculations using a plane-wave basis set," Phys. Rev. B, vol. 54, no. 16, pp. 11169-11186, May 1996. 
[11] S. Birner, S. Hackenbuchner, M. Sabathil, G. Zandler, J. A. Majewski, T. Andlauer, T. Zibold, R. Morschl, A. Trellakis, and P. Vogl, "Modeling of semiconductor nanostructures with nextnano ${ }^{3}$." Acta Phys. Polon. A, vol. 110, pp. 111-124, Aug. 2006.

[12] J. L. Lyons, A. Janotti, and C. G. Van de Walle, "Effects of hole localization on limiting $p$-type conductivity in oxide and nitride semiconductors" J. Appl. Phys., vol. 115, no. 1, pp. 012014-1-012014-7, Jan. 2014.

[13] J. L. Lyons, A. Janotti, and C. G. Van de Walle, "Shallow versus deep nature of $\mathrm{Mg}$ acceptors in nitride semiconductors," Phys. Rev. Lett., vol. 108, pp. 156403-1-156403-5, Apr. 2012.

[14] A. Castiglia, J.-F. Carlin, and N. Grandjean, "Role of stable and metastable $\mathrm{MgH}$ complexes in $p$-type $\mathrm{GaN}$ for cw blue laser diodes," Appl. Phys. Lett., vol. 98, pp. 213505-1-213505-3, May 2011. 